\title{
De Sanctis e la storiografia letteraria italiana
}

\author{
Roberto Antonelli \\ Università di Roma «La Sapienza»
}

\begin{abstract}
L'autore analizzza l'impatto della «Storia della letteratura italiana» di Francesco De Sanctis sulla storiografia italiana del XX secolo, rilevando come la linea storicistica «lineare» di De Sanctis sia stata assunta come modello storiografico di tutte le Storie letterarie successive ma come nel contempo sia stata oggetto anche di una riduzione ideologica, da Croce a Gentile, a Gramsci, con la sottolineatura degli aspetti «militanti» della sua opera. De Sanctis ha concepito la sua «Storia» in funzione del processo unitario risorgimentale: soltanto Carlo Dionisotti ha criticato le ragioni storiche di tale modello proponendone acutamente uno radicalmente alternativo, di tipo geografico-storico,che recentemente ha iniziato a produrre i suoi risultati, anche a livello formativo.
\end{abstract}

Parole-chiave: De Sanctis; Storiografia letteraria; Croce; Geografia e storia; Dionisotti.

\begin{abstract}
The author analyses the impact of the «Storia della letteratura italiana» by Francesco De Sanctis about the Italian historiography of the Twentieth century, pointing out how the historicist line by De Sanctis has established itself as a historiographical model for the whole later literary history while, at the same time, it proposes an ideological reduction, from Croce and Gentile, to Gramsci, with the underlining of the «militant» aspects of his work. De Sanctis has conceived his "Storia» in relationship with the unitary process of the Risorgimento: only Carlo Dionisotti has criticised the historical reasons of such a model, and has aptly proposed a radical alternative one of a historical-geographical type. This alternative has recently begun to achieve results at a formative level.
\end{abstract}

Keywords: De Sanctis; Literary historiography; Croce; Geography and history; Dionisotti. 
Da De Sanctis la cultura letteraria italiana eredita due grandi problemi: 1. la storia della letteratura come storia della civiltà, per di più finalistica, e, conseguentemente, la questione del modello storiografico; 2) il rapporto fra testo letterario e storia, fra «letterarietà» e storia o, per dirla con la vulgata (che peraltro Contini già nel 1949 ha mostrato insufficiente, vista la variabilità terminologica desanctisiana), tra forma e contenuto.

Per quel che riguarda la prima questione, in un passo notissimo De Sanctis sottolinea quanto una storia della letteratura debba alle ricerche specialistiche e quanto, nel momento in cui stava scrivendo la sua Storia della letteratura, ancora rimanesse da fare, arrivando paradossalmente a sottolineare che quello era il tempo delle monografie:

Una storia della letteratura è come l'epilogo, l'ultima sintesi di un immenso lavoro di tutta intiera una generazione nelle singole parti. Tiraboschi, Andrés, Ginguené sono sintesi del passato. Oggi tutto è rinnovato, da tutto sbuccia un nuovo mondo: filosofia, critica, arte, storia, filologia. Non ci è più alcuna pagina della nostra storia che resti intatta. Dovunque penetra con le sue ricerche lo storico e il filologo, e con le sue speculazioni il filosofo e il critico. L'antica sintesi è sciolta. Ricomincia il lavoro paziente dell'analisi, parte per parte. Quando una storia della letteratura sarà possibile? Quando questo lavoro paziente avrà portata la sua luce in tutte le parti; quando su ciascuna epoca, su ciascun scrittore importante ci sarà tale monografia o studio o saggio che dica l'ultima parola e sciolga tutte le quistioni. Il lavoro di oggi non è la storia, ma è la monografia, ciò che i Francesi chiamano uno studio. ${ }^{1}$

De Sanctis ha quindi ben chiaro che la sua Storia è un po' particolare: è il frutto di una particolare congiuntura storica e che l'Italia ha una filosofia e una letteratura «la quale ha la sua leva fuori di lei», nella libertà e nella nazionalità, quanto a dire nella politica:

L'Italia è stata finora avviluppata come di una sfera brillante, la sfera della libertà e della nazionalità, e n’è nata una filosofia e una letteratura, la quale ha la sua leva fuori di lei, ancorché intorno a lei. Ora si dee guardare in seno, dee cercare sé stessa; la sfera dee svilupparsi e concretarsi come sua vita interiore. [...] Viviamo molto sul nostro passato e del lavoro altrui. Non ci è vita nostra e lavoro nostro. E da' nostri vanti s'intravede la coscienza della nostra inferiorità. Il grande lavoro del secolo decimonono è al suo termine. Assistiamo a una nuova fermentazione d'idee, nunzia di una nuova formazione. Già vediamo in questo secolo disegnarsi il nuovo secolo. E questa volta non dobbiamo trovarci alla coda, non a' secondi posti. ${ }^{2}$

In questo senso l'intreccio fra spinta storico-politica risorgimentale e scrittura della sua Storia formano un tutt'uno. Ricostruzione delle basi letterarie

1. F. De Sanctis, Settembrini e i suoi critici (1869), poi in Nuovi saggi critici (1879), Napoli $1890^{4}$, p. $251-252$.

2. F. De Sanctis, Storia della letteratura italiana, a c. di N. Gallo, intr. di N. Sapegno, Con una nota introduttiva di C. Muscetta, Torino 1975, 2 voll., II, p. 975. 
dell'unità d'Italia e Risorgimento si collocano in un processo ascendente che trova la sua soluzione con la presa di Roma: la sua è una storia particolare, una storia militante della letteratura, finalizzata:

Il fondamento scientifico di questo mondo è la cosa effettuale, come te la porge l'esperienza e l'osservazione. L'immaginazione, il sentimento, l'astrazione sono così perniciosi nella scienza, come nella vita. Muore la scolastica, nasce la scienza. Questo è il vero machiavellismo, vivo, anzi giovane ancora. E' il programma del mondo moderno, sviluppato, corretto, ampliato, più o meno realizzato. E sono grandi le nazioni che più vi si avvicinano. Siamo dunque alteri del nostro Machiavelli. Gloria a lui quando crolla alcuna parte dell'antico edificio. E gloria a lui quando si fabbrica alcuna parte del nuovo. In questo momento che scrivo, le campane suonano a distesa, e annunziano l'entrata degl'italiani a Roma. Il potere temporale crolla. E si grida il viva all'unità d'Italia. Sia gloria a Machiavelli. ${ }^{3}$

Sarebbe però certo parziale giudicare la Storia desanctisiana, come fosse una storia dettata da interessi puramente politici: storiografia e valutazione estetica si compongono in lui, e nella sua opera, in un insieme non scomponibile. E' qui che si colloca la seconda questione lasciata in eredità dalla sua opera, quella della definizione estetica dell'opera letteraria e, conseguentemente, del suo rapporto con la storia letteraria, in particolare con una storia della letteratura come quella da lui costruita. Se infatti prima della stesura della Storia, nel 1858, la letteratura e la sua storia hanno un senso, è per il tipo particolare di rapporto che intercorre fra contenuto e forma ( $L$ '«idea» e l'estetica dello Hegel: «ogni contenuto è una totalità, che come idea appartiene alla scienza, come esistere materiale appartiene alla realtà, come forma appartiene all'arte [...] Il mistero della vita è che il tutto non comparisce mai come tutto, ma come parte, la quale non esclude ma si assimila il rimanente»). Concetto ribadito e analiticamente articolato e sviluppato ancora nel 1869, a Storia quasi completata, e basato, seppur con qualche rielaborazione, sull'insegnamento hegeliano:

La forma non è a priori, non è qualcosa che stia da sé e diversa dal contenuto, quasi ornamento o veste o apparenza o aggiunto di esso, anzi è essa generata dal contenuto, attivo nella mente dell'artista: tal contenuto tal forma. E il contenuto è attivo, appunto perché ha la sua poesia, il suo bello naturale, come la natura ha il suo bello reale, ha qualcosa di proprio che fa impressione e mette in movimento il cervello dell'artista, ed apparisce nella forma. Ivi, nella forma, il critico ritrova il contenuto, «da lui già esaminato come un antecedente», lo ritrova non più natura, ma arte; non più qual era, ma qual è divenuto, e sempre tutto esso, col suo valore, colla sua importanza. [...] Il contenuto non è dunque indifferente, non è trascurato. Apparisce due volte nella nuova critica: la prima, come naturale e astratto, qual era; la seconda, come forma, qual è divenuto. [...] Il contenuto è sottoposto a tutte le vicende della storia; nasce e muore; la forma è immortale. ${ }^{4}$

3. Ibid., p. 606-607.

4. F. De Sanctis, Settembrini, cit., p. 240. 
dell'unità d'Italia e Risorgimento si collocano in un processo ascendente che trova la sua soluzione con la presa di Roma: la sua è una storia particolare, una storia militante della letteratura, finalizzata:

Il fondamento scientifico di questo mondo è la cosa effettuale, come te la porge l'esperienza e l'osservazione. L'immaginazione, il sentimento, l'astrazione sono così perniciosi nella scienza, come nella vita. Muore la scolastica, nasce la scienza. Questo è il vero machiavellismo, vivo, anzi giovane ancora. E' il programma del mondo moderno, sviluppato, corretto, ampliato, più o meno realizzato. E sono grandi le nazioni che più vi si avvicinano. Siamo dunque alteri del nostro Machiavelli. Gloria a lui quando crolla alcuna parte dell'antico edificio. E gloria a lui quando si fabbrica alcuna parte del nuovo. In questo momento che scrivo, le campane suonano a distesa, e annunziano l'entrata degl'italiani a Roma. Il potere temporale crolla. E si grida il viva all'unità d'Italia. Sia gloria a Machiavelli. ${ }^{3}$

Sarebbe però certo parziale giudicare la Storia desanctisiana, come fosse una storia dettata da interessi puramente politici: storiografia e valutazione estetica si compongono in lui, e nella sua opera, in un insieme non scomponibile. E' qui che si colloca la seconda questione lasciata in eredità dalla sua opera, quella della definizione estetica dell'opera letteraria e, conseguentemente, del suo rapporto con la storia letteraria, in particolare con una storia della letteratura come quella da lui costruita. Se infatti prima della stesura della Storia, nel 1858, la letteratura e la sua storia hanno un senso, è per il tipo particolare di rapporto che intercorre fra contenuto e forma ( $L$ '«idea» e l'estetica dello Hegel: «ogni contenuto è una totalità, che come idea appartiene alla scienza, come esistere materiale appartiene alla realtà, come forma appartiene all'arte [...] Il mistero della vita è che il tutto non comparisce mai come tutto, ma come parte, la quale non esclude ma si assimila il rimanente»). Concetto ribadito e analiticamente articolato e sviluppato ancora nel 1869, a Storia quasi completata, e basato, seppur con qualche rielaborazione, sull'insegnamento hegeliano:

La forma non è a priori, non è qualcosa che stia da sé e diversa dal contenuto, quasi ornamento o veste o apparenza o aggiunto di esso, anzi è essa generata dal contenuto, attivo nella mente dell'artista: tal contenuto tal forma. E il contenuto è attivo, appunto perché ha la sua poesia, il suo bello naturale, come la natura ha il suo bello reale, ha qualcosa di proprio che fa impressione e mette in movimento il cervello dell'artista, ed apparisce nella forma. Ivi, nella forma, il critico ritrova il contenuto, «da lui già esaminato come un antecedente», lo ritrova non più natura, ma arte; non più qual era, ma qual è divenuto, e sempre tutto esso, col suo valore, colla sua importanza. [...] Il contenuto non è dunque indifferente, non è trascurato. Apparisce due volte nella nuova critica: la prima, come naturale e astratto, qual era; la seconda, come forma, qual è divenuto. [...] Il contenuto è sottoposto a tutte le vicende della storia; nasce e muore; la forma è immortale. ${ }^{4}$

3. Ibid., p. 606-607.

4. F. De Sanctis, Settembrini, cit., p. 240. 
Sul rapporto fra contenuto e forma Croce nell' Estetica pervenne, estremizzandolo, a risultati diversi, con implicazioni notevoli anche riguardo alla natura della storia letteraria il cui senso, come è noto, è negato in radice:

La conoscenza ha due forme: o è conoscenza intuitiva o è conoscenza logica; conoscenza per la fantasia o conoscenza per l'intelletto; conoscenza dell'individuale o dell'universale; [...] è, insomma, o produttrice d'immagini o produttrice di concetti. [...]

Ogni vera intuizione o rappresentazione è, insieme, espressione. Ciò che non si oggettiva in una espressione non è intuizione o rappresentazione, ma sensazione o naturalità. [...] Intuire è esprimere; e nient'altro (niente di più, ma niente di meno) che esprimere.

[...] [Materia e forma] sono non già due atti nostri, di cui l'uno stia di fronte all'altro, ma l'uno è un di fuori che ci assalta e ci trasporta, l'altro è un di dentro che tende ad abbracciare quel di fuori e a farlo suo. La materia, investita e trionfata dalla forma, dà luogo alla forma concreta. E' la materia, è il contenuto quel che differenzia una nostra intuizione da un'altra: la forma è costante, l'attività spirituale; [...]

[...] La materia poetica corre negli animi di tutti: solo l'espressione, cioè la forma, fa il poeta. E qui si trova la verità della tesi che nega all'arte qualsiasi contenuto, intendendosi per contenuto appunto il concetto intellettuale. In questo senso, posto «contenuto» uguale a "concetto», è esattissimo non solo che l'arte non consiste nel contenuto, ma che essa non ha contenuto.

[...] Se l'espressione è forma della coscienza, come cercare l'origine storica di ciò che non è prodotto della natura, e che della storia umana è presupposto?

Il giudizio che conseguentemente lo stesso Croce dà della Storia desanctisiana $^{6}$ si muove su due piani; da una parte ne esalta il valore di storia, si badi, di «tutta la vita italiana, religiosa politica morale», senza mai usare il termine «letteraria», dall'altra ne pone in rilievo lo stile, dunque la valenza estetica, del tutto conseguentemente con le premesse teoriche crociane ("estetica come scienza dell'espressione»), che portano ad una storia quale «opus rhetoricum maxime»: "Qui importa considerare l'animo di lui, il sentimento che alita nei suoi scritti, lo stile che ne è l'espressione». Della soluzione desancisiana del rapporto fra contenuto-forma e storiografia letteraria non c'è più traccia esplicita, ${ }^{7}$ implicitamente acquisendo De Sanctis all'interno del proprio sistema. La Storia della letteratura non può valere sul piano del giudizio estetico (ma essere semmai oggetto, in quanto opera d'arte, di un giudizio estetico).

5. B. Croce, Estetica come scienza dell'espressione e linbguistica generale (1902), Bari 1958, p. 3 e segg.

6. ID., Francesco De Sanctis, in La letteratura della nuova Italia. Saggi critici, Bari 1921, p. 357-377.

7. Ibid., pur se sottolineava la grande novità di un critico «nel quale il giudizio sull'opera d'arte diventava al tempo stesso libero da ogni dominio estraneo e rigidamente sottomesso al criterio dell'arte». 
Con la posizione crociana consuona ovviamente Borgese, nel suo periodo crociano, e la sua posizione ha valore abbastanza rappresentativo di un intero filone critico egemone per decenni:

Ora il De Sanctis diede un esemplare mirabile [...] di una storia della civiltà mostrata per via della letteratura. Si ripensi al filo d'Arianna di quell'opera: il popolo italiano, che dal forte spiritualismo di Dante e dall'ingenua fede del Cavalca e del Compagni passava, col Petrarca, ad un torbido atteggiamento dei sensi della realtà della ragione: che, improvvisamente affacciatosi alla vita civile, si ride e si burla della barbarie plebea e chiericale, la quale rimaneva vestigio del forte medio evo negli uomini indotti di greco e di latina. Ride con indifferenza nel Boccaccio, con scetticismo nell'Ariosto, con cinismo nel Folengo; la civiltà si muta in raffinatezza impotente e indolente, e distrugge nella coscienza e nella letteratura ogni serietà di contenuto; conduce all'uomo del Guicciardini e alla poesia del Marino, mentre d'altro canto prepara, nel sottosuolo, la nuova scienza, che darà in progresso di tempo il nuovo poeta ed il nuovo uomo. Or questa era certamente continuità di tela, e il De Sanctis non è da riprovare perché trascurò la folla dei mediocri (che pure seppe in qualche modo aggruppare o additare intorno ai maggiori), essendosi egli proposto di mostrar nella letteratura lo specchio della vita civile e giovandogli per tale scopo massimamente quegli scrittori, che aveano conseguito la massima potenza nella espressione dei loro tempi. Era il piú perfetto modello di storia letteraria? Forse i nostri desiderii son mutati; [...] forse noi prepariamo un nuovo ideale di storia letteraria; ma di quello che fu in mente ai romantici, dal Berchet al Cantú, l'opera del De Sanctis è un capolavoro di esecuzione. ${ }^{8}$

Croce dunque quale intelligente e tendenzioso lettore di De Sanctis, del quale comunque si pone come continuatore, iscrivendolo in un disegno strategico di costruzione dell'ideologia borghese della Nuova Italia. In tal senso quella di Croce è presunzione in gran parte legittima, anche per la grandiosità del suo progetto postrisorgimentale, concepito come completamento liberale di quello risorgimentale, fino ad includere il disegno desanctisiano in un quadro teorico generale: la filosofia dello spirito.

Il primo ad autorizzare del resto tale ovvia ma parziale interpretazione, con sottolineature necessariamente più "contenutistiche», è proprio De Sanctis: si rileggano le affermazioni contenute nella Storia della letteratura vs quelle consegnate precedentemente ai Saggi critici (non per nulla saggi monografici, e come tali apparentemente più utilizzabili da Croce poiché più vicini al suo sistema): è lo stesso De Sanctis che per primo sostiene - con molta consapevolezza, e perfino modestia - una lettura della Storia della letteratura come storia di una civiltà, alla luce del Risorgimento.

A queste ragioni è forse dovuto lo scarso appeal di De Sanctis come critico, fuori d'Italia. Passione risorgimentale e urgenza di offrire alla "Nuova Italia» un quadro del recente passato risorgimentale e del suo senso: «storia» e «senti-

8. G. A. Borgese, Storia della critica romantica in Italia, in F. De SANCTIS, Storia della letteratura italiana, a c. di N. Gallo, «Introduzione» di G. Ficara, Torino: Einaudi-Gallimard, 1996, Sulla «letteratura italiana» di F. De Sanctis, Antologia critica a c. di G. Ficara, p. 820. 
mento» formano un insieme che dà conto dei giudizi di Croce e di tanti dopo di lui, fino a Wellek ${ }^{9}$, compresa la sottolineatura della «narratività» del testo desanctisiano, già in Croce ma ancor più in Debenedetti e ancora recentemente presso Sanguineti e Ficara, fino a concepirlo, oggi, come una sorta di grande romanzo storico: il capolavoro del Romanticismo risorgimentale italiano, un po' come Verdi in musica. ${ }^{10}$ Lettura certamente legittima e acuta, ma in qualche modo limitativa della personalità di De Sanctis.

L'uso "politico»(-culturale) di De Sanctis inizia dunque con Croce e la sua battaglia, liberale e antisocialista, contro il positivismo e contro la scuola storica $^{11}$ : De Sanctis è esaltato come risorgimentale e precursore dell'idealismo, oltre che come serbatoio di temi (ivi compresa l'Estetica), suggestioni critiche e perfino formule linguistiche.

Mentre l'interpretazione tendenziosa crociana insisteva su un filo in qualche modo continuo, con Gentile, a regime fascista ormai trionfante, si determina una vera e propria svolta, in evidente polemica con Croce (ribadita poi più esplicitamente in La filosofia dell'arte), di cui inizialmente si discutono i presupposti teorici, rilevando come la filosofia di riferimento di De Sanctis, l'hegelismo, non fosse riducibile al sistema crociano (che «non è filosofia, ma semplice descrizione empirica e dommatica»). De Sanctis è innanzitutto il critico e lo storico di «una cultura dell'animo e riforma della vita», è innanzitutto il maestro di una critica militante perfettamente adatta a un momento in cui «oggi [...], almeno in Italia, si ride delle preoccupazioni ingenue e melense per la purezza degli ideali dei "chierici"», poiché «la stessa arte, la stessa filosofia non si sanno più concepire se non in funzione della vita, e cioè come forme della stessa vita che si svolge sotto l'impero d'una stessa legge nella politica come nella scuola, nelle armi come negli studi, nel lavoro come nella riflessione scientifica». Non c'è più posto per l'«arte, pura arte, poesia in opposizione alla prosa, forma da definire prescindendo dal contenuto, ecc.». Bisogna «tornare a De Sanctis»:

9. René WeLLEK però, mentre sottolineava la scarsa conoscenza dell'opera di De Sanctis fuori d'Italia, esaltava anche la sua capacità di «fondere felicemente un ampio schema storico con una critica rigorosa, la teoria con la pratica, la generalizzazione estetica con l'analisi specifica», fino a definirla «la più bella storia letteraria che sia mai stata scritta» (A History of Modern Criticism (1965) [tr. it. Storia della critica moderna (1750-1950), Bologna 19581969, 5 voll., IV, p. 155]).

10. Su tali aspetti cfr. l'Introduzione di G. Ficara a F. De SANCTIS, Storia della letteratura cit., p. xv e segg.

11. La funzione con cui Croce utilizzò la Storia desanctisiana è perfettamente chiara a Carlo DionisotTi, Postilla a una «lettera scarlatta» (1946), poi in Geografia e storia della letteratura italiana (1967), Torino 1977, p. 19: «Nel dominio della letteratura italiana, conteso a quel tempo dai carducciani e dai filologi, come allora si diceva, di scuola tedesca, in un reggimento oligarchico fondato sulle cattedre universitarie e su riviste spcializzate, il Croce non ebbe da fare breccia con la prepotenza della conquista [...]. Perno del rivolgimento la Storia del De Sanctis: e il nuovo assetto fu raggiunto sulla base di una teocrazia desanctisiana alla quale si adattarono anche i precedenti oligarchi, accettando per sé funzioni più definite e controllate». 
Il vero è che l'estetica del De Sanctis è non inquadrata — come oggi barbaricamente si dice- ma fondata in una filosofia; e perciò è tutt'uno con questa filosofia; laddove l'estetica dei continuatori (voglio dire, di quelli che si presumono o sono ritenuti suoi continuatori) non ha questo fondamento, perché non è filosofia, ma semplice descrizione empirica e dommatica. [...] Oggi che tra gl'Italiani s'è diffusa la convinzione che una cultura dell'intelligenza che non sia cultura dell'animo e riforma della vita è una cultura vuota e falsa; oggi che la stessa arte, la stessa filosofia non si sanno più concepire se non in funzione della vita, e cioè come forme della stessa vita che si svolge sotto l'impero d'una stessa legge nella politica come nella scuola, nelle armi come negli studi, nel lavoro come nella riflessione scientifica; oggi che, almeno in Italia, si ride delle preoccupazioni ingenue e melense per la purezza degli ideali dei «chierici»; oggi nella critica letteraria, e non soltanto in essa, bisogna tornare a De Sanctis. E' tempo di spazzare i ragnateli di quella inafferrabile critica che pretende invano di dividere l'indivisibile e fissare un momento ideale della vita dello spirito: arte, pura arte, poesia in opposizione alla prosa, forma da definire prescindendo dal contenuto, ecc. L'arte che si potesse così additare, in sé e per sé, sarebbe in verità un alibi assurdo per l'uomo. Il quale sempre che ha vissuto una schietta forma d'arte, ha sentito dentro di essa tutta la sua umanità, con la sua fede e con la sua passione; da cui nessuno s'è potuto mai staccare se non per precipitare nel falso della vita e insieme dell'arte. ${ }^{12}$

Gramsci raccoglie immediatamente la "parola d'ordine» gentiliana, per capovolgerne il segno, pur se sulla definizione del tipo di atteggiamento critico di De Sanctis, in quanto appassionato, «aperto alla vita» e non "frigido» i due, da sponde opposte, concordano: Gramsci è contro l'interpretazione dei De Sanctis, militante e "fascista», di Gentile, ma è allo stesso tempo contro quella «liberale» di Croce (di cui coglie la tendenziosità, ma in modo più acuto, o corretto, di Gentile: «Il Croce riesce a distinguere questi aspetti diversi del critico, che nel De Sanctis erano organicamente uniti e fusi»), rivendicando invece, in un brano famoso e poi citatissimo, che varrà la pena di rileggere per intero, la complessità delle posizioni De Sanctis e la sua fisionomia sostanzialmente democratica, non liberale:

Cosa significa e cosa può e dovrebbe significare la parola d'ordine di Giovanni Gentile «Torniamo al De Sanctis!»? Significa «tornare» meccanicamente ai concetti che il De Sanctis svolse intorno all'arte e alla letteratura, o significa assumere verso l'arte e la vita un atteggiamento simile a quello assunto dal De Sanctis ai suoi tempi? Posto questo atteggiamento come "esemplare», è da vedere: 1) in che sia consistita tale esemplarità; 2) quale atteggiamento sia oggi corrispondente, cioè quali interessi intellettuali e morali corrispondano oggi a quelli che dominarono l'attività del De Sanctis e le impressero una determinata direzione. Né si può dire che la biografia del De Sanctis, pur essendo essenzialmente coerente, sia stata "rettilinea», come volgarmente s'intende. Il De

12. G. Gentile, Torniamo a De Sanctis!, in Quadrivio, 6 agosto 1933 [rist. in Memorie italiane, Firenze, 1936, p. 173-181]. 
Sanctis, nell'ultima fase della sua vita e della sua attività, rivolse la sua attenzione al romanzo «naturalista» o "verista» e questa forma di romanzo, nell'Europa occidentale, fu l'espressione «intellettualistica» del movimento piú generale di "andare al popolo», di un populismo di alcuni gruppi intellettuali sullo scorcio del secolo scorso, dopo il tramonto della democrazia quarantottesca e l'avvento di grandi masse operaie per lo svíiluppo della grande industria urbana. Del De Sanctis è da ricordare il saggio La scienza e la vita, il suo passaggio alla Sinistra parlamentare, il suo timore di tentativi forcaioli velati da forme pompose, ecc.

Un giudizio del De Sanctis: «Manca la fibra perché manca la fede. E manca la fede perché manca la cultura». Ma cosa significa "cultura» in questo caso? Significa indubbiamente una coerente, unitaria e di diffusione nazionale «concezione della vita e dell'uomo», una "religione laica», una filosofia che sia diventata appunto "cultura", cioè abbia generato un'etica, un modo di vivere, una condotta civile e individuale. Ciò domandava innanzi tutto l'unificazione della «classe colta» e in tal senso lavorò il De Sanctis con la fondazione del Circolo filologico, che avrebbe dovuto determinare «1'unione di tutti gli uomini colti e intelligenti» di Napoli; ma domandava specialmente un nuovo atteggiamento verso le classi popolari, un nuovo concetto di ciò che è «nazionale», diverso da quello della Destra storica, più ampio, meno esclusivista, meno "poliziesco", per così dire.

[...] La critica del De Sanctis è militante non frigidamente estetica, è la critica di un periodo di lotte culturali, di contrasti fra concezioni di vita antagonistiche. Le analisi del contenuto, la critica della «struttura" delle opere, cioè della coerenza logica e storico-attuale delle masse di sentimenti rappresentati artisticamente, sono legate a questa lotta culturale: proprio in ciò pare consista la profonda umanità e l'umanesimo del De Sanctis, che rendono tanto simpatico anche oggi il critico. Piace sentire in lui il fervore appassionato dell'uomo di parte, che ha saldi convincimenti morali e politici e non li nasconde e non tenta neanche di nasconderli. Il Croce riesce a distinguere questi aspetti diversi del critico, che nel De Sanctis erano organicamente uniti e fusi.

[...] Insomma, il tipo di critica letteraria propria della filosofia della prassi è offerto dal De Sanctis, non dal Croce o da chiunque altro (meno che mai dal Carducci): essa deve fondere la lotta per una nuova cultura, cioè per un nuovo umanesimo, la critica del costume, dei sentimenti e delle concezioni del mondo, con la critica estetica o puramente artistica nel fervore appassionato, sia pure nella forma del sarcasmo». ${ }^{13}$

Nel dopoguerra, a Quaderni dal carcere ormai noti (Gli intellettuali e l'organizzazione della cultura è del 1949), la posizione di Gramsci viene assunta come paradigma assoluto della politica culturale del Partito comunista italiano e di moltissimi critici che al marxismo si ispirano, fino a determinare nella critica militante una vera e propria tipologia critica, come forse Gianfranco Contini presagisce (o già rileva?), proprio nel 1949, quando ancora non è stata pubblicata Letteratura e vita nazionale, con leggerezza ma con distacco critico ben evidente nei riguardi di De Sanctis: 
Parlare di Francesco De Sanctis è diventato singolarmente difficile da quando, nella considerazione popolare il mito e il tipo hanno surrogato l'uomo. [...] Cosa più preoccupante, egli non appare però ai molti un critico, bensì il critico;

[...] Ogni visione teologica, o meglio emanatistica, della poesia, nella quale ogni universo poetico appare necessariamente integrato dal successivo, per definizione si fonda sulla nozione del progresso in poesia. Anche il De Sanctis [...] crede al progresso [...] seppure lo sposti verso le «forme», opponendole al "genio». ${ }^{14}$

La posizione di Gramsci è utilizzata dalla critica di parte comunista soprattutto quale critica nei confronti di Croce, per tentare di rovesciarne l'egemonia esercitata sulla cultura e in particolare sulla critica letteraria, tanto che ancora nel 1967, nella Premessa e dedica ad un libro d'importanza epocale per la storiografia letteraria italiana, Geografia e storia della letteratura italiana, Carlo Dionisotti ritiene necessario reintervenire sulla questione, ritenendola ancora viva, specie per un libro il cui saggio fondativo era stato letto come prolusione nel 1949 e stampato nel 1951:

Poiché dopo la guerra si è sviluppata una diffusa e acre insofferenza del governo che lungamente, per quasi cinquant'anni, Benedetto Croce esercitò sulla cultura italiana, e perché negli scritti qui raccolti una qualche riserva è espressa nei riguardi di quel governo, [...] mi sia lecito dire alla buona e chiaramente che, se per assurda ipotesi la scelta si ponesse, oggi, fra la lezione del De Sanctis e quella del Croce, non esiterei, come studioso di storia e di letteratura italiana, un istante: ancora starei umilmente e volentieri, come mi pregio di essere stato sempre, col più formidabile lettore e intenditore di testi italiani che a mia notizia sia apparso dal Settecento a oggi. ${ }^{15}$

Potremmo dire, se volessimo semplificare su un piano storico-politico, che però sul momento vince la lettura gramsciana perché nello scontro in atto anche in Italia, in piena guerra fredda, sul terreno cultural-letterario vince il PCI e perde la borghesia democratica, il Partito d'Azione (azionisti erano sia Contini, cattolico, che Dionisotti, laico, fra loro amici e reciproci estimatori). Insomma, dopo il recupero crociano, è il ventennio di dittatura fascista, la Seconda guerra mondiale e la politica del dopoguerra che segnano anche la storiografia e la teoria letteraria.

Ciò coinvolge anche le posizioni storiografiche elaborate in questi anni (1949/1951) dallo stesso Dionisotti, in aperto e radicale rovesciamento delle tesi di De Sanctis, non tanto riguardo alla funzione e alle modalità della critica, ma proprio dell'interpretazione desanctisiana e risorgimentale della storia italiana e quindi del modello strutturale della Storia:

Poco prima dell'ultima guerra tornò in discussione fra studiosi italiani il quesito se e fino a qual segno la storia d'Italia potesse dirsi unitaria. In tale occasione Benedetto Croce pubblicò [...] un saggio in cui ribadiva la tesi, coerente

14. G. ConTINI, Introduzione a F. De Sanctis, Scritti critici, 1949, ora in ID., Varianti e altra linguistica. Una raccolta di saggi (1938-1968), Torino: Einaudi, 1970, p. 499.

15. C. DionisotTI, Geografia e storia, cit., p. 11. 
al suo pensiero, ma splendidamente ardita in quella sede e a quella data (1936), che di una storia d'Italia anteriore al processo unitario del Risorgimento non fosse il caso di parlare, risolvendosi essa nella varia storia delle singole unità politiche, regionali o municipali o altramente costituite, in cui l'Italia per secoli era stata divisa. ${ }^{16}$

Lo schema per Dionisotti andava invertito, poiché si giustificava allora, dopo la guerra, «un riesame della questione» per vedere "fino a qual punto fosse accettabile la linea unitaria comunemente seguita nel disegno storico della letteratura italiana». ${ }^{17}$ Dunque, non "storia della letteratura italiana» ma "geografia e storia della letteratura italiana»: "Si può discutere se quel che in una letteratura più importa, l'offerta che essa reca di umana poesia, soffra o no distinzioni e definizioni di spazio e di tempo. Ma discutibile non sembra il principio che, ove a tali distinzioni e definizioni per qualunque motivo si ricorra, esse debbano farsi avendo riguardo alla geografia e alla storia, alle condizioni che nello spazio e nel tempo stringono ed esaltano la vita degli uomini» ${ }^{18}$, giusta l'osservazione crociana del 1936, da cui Dionisotti era partito per iniziare la prolusione londinese del 1949.

Dionisotti con Croce dunque, malgrado le «riserve» talvolta espresse, contro Gramsci e quindi contro De Sanctis, così come del resto Contini con Croce: su presupposti diversi ma uniti nel rifiuto di quell'egemonia culturale gramsciana che sembrava intaccare l'autonomia della letteratura, sia sul piano storiografico che valoriale. ${ }^{19}$ Dionisotti metteva in discussione i presupposti storiografici della Storia della letteratura di De Sanctis; Contini, in un'analisi linguistica ravvicinata dell'uso desanctisiano, ne discuteva i presupposti estetici, condensati nell'uso del binomio «forma e contenuto" (ovvero le due questioni lasciate in eredità da De Sanctis, dalle quali siamo partiti), per storicizzarne il senso e per proporre un diverso recupero e analisi della forma. ${ }^{20} \mathrm{Sul}$ piano storiografico era certo più vicina alla strategia crociana la serie La Letteratura italiana. Storia e testi della Ricciardi, diretta da Raffaele Mattioli, Pietro Pancrazi e Alfredo Schiaffini, che non a caso si apriva con la pubblicazione di un volume antologico di scritti crociani (nel piano dell'opera, ultimo della serie), curato dallo stesso autore, e che dei volumi di storia pubblicherà soltanto Il Trecento di Natalino Sapegno, forse anche in ragione dell'uscita, nel 1965, della Storia della Letteratura Italiana della Garzanti, diretta dallo stesso Sapegno e da Emilio Cecchi, secondo l'antico modello «per secoli» della Storia letteraria d'Italia della Casa editrice Vallardi «Scritta da una Società di Professori».

16. Ibid., p. 25.

17. Ibid., p. 35.

18. Ibid., p. 54.

19. E uniti, a ben vedere, anche nella valorizzazione dell'importanza della varietà dei centri italiani: sul piano strettamente linguistico in Contini, su quello storico-culturale in Dionisotti. E uniti anche nel recupero dell'importanza della scuola storica.

20. Si veda G. ContinI, Introduzione a De Sanctis cit., p. 499-531, d'importanza paragonabile al saggio dello stesso Contini sull'influenza culturale di Benedetto Croce. 
A Sapegno si dovevano già i volumi del Compendio di storia della letteratura italiana per le scuole medie superiori, un vero e proprio classico della storiografia letteraria italiana (formerà varie generazioni di giovani), uscito a partire dal 1938; nel 1947, con il terzo volume, Dal Foscolo ai moderni, Sapegno tenterà di fondere la lezione crociana e quella desanctisiana, secondo una prospettiva storiografica che maturerà pienamente proprio nell'impresa della Storia della Letteratura Italiana Garzanti, dove verrà ancora una volta pienamente confermato il modello storiografico, non ideologico, di De Sanctis, e la garbata, come di consueto, ma decisa ripulsa di ogni altra prospettiva:

I presupposti teorici, i criteri di metodo, gli strumenti tecnici idonei all'elaborazione di una seria storiografia letteraria si proposero come oggetto di una analisi approfondita e di un dibattito esteso e fruttuoso soltanto nel corso del secolo xix, nel quadro dello storicismo romantico; e proprio in Italia tale dibattito attinse forse alle sue formulazioni più sottili e rigorose, ovviamente in rapporto con il rapido e generoso sviluppo della rivoluzione nazionale e borghese e con lo sforzo che essa doveva comportare, da parte dei gruppi dirigenti e degli intellettuali che ne interpretavano le aspirazioni, di un'organica presa di coscienza delle varie vicende nel tempo e della struttura coerente e unitaria della nostra cultura.

Non soltanto allora fu concordemente rifiutata l'idea di una storia letteraria come mero repertorio di notizie biografiche e bibliografiche, quale era stata fissata in teoria ed in pratica dalla pur benemerita erudizione settecentesca; sì anche divenne oggetto di discredito l'altra tendenza, che a quella in parte si era contrapposta e in parte accompagnata quasi a guisa di integrazione e di complemento, a fornire un parallelo repertorio di marginali rilievi grammaticali e rettorici. D'altra parte la ricerca di un piano di discorso più elevato ed organico, più intrinsecamente storico, attraverso le discussioni a cui in vario modo parteciparono le maggiori personalità del mondo intellettuale (dal Foscolo ai redattori del "Conciliatore» e dell' «Antologia», dal Mazzini al Gioberti e al Cattaneo, dal Tommaseo al Tenca e all'Imbriani) e attraverso i primi tentativi di sistemazione espositiva (del Maffei e dell'Emiliani Giudici, del Cantù e del Settembrini) sulla scia di frettolosi schemi politici e moralistici, doveva presto rivelarsi infruttuosa e insoddisfacente al paragone di un concetto dell'arte che veniva prendendo coscienza di se stesso sul piano speculativo, coscienza voglio dire della propria natura distinta e della propria autonomia.

A questo punto venne ad inserirsi nel dibattito ancora aperto il De Sanctis, con la sua travagliata consapevolezza teorica e con il concreto esempio della sua attività di storico. Vera storia della letteratura non poteva essere, a parer suo, né una valutazione di contenuti astratti, di mondi intenzionali, di per sé anteriori al momento della creazione estetica, né un'indagine altrettanto astratta del «lato apparente e superficiale della forma» che si suol definire come stile. Né quella materia morale, né quegli strumenti espressivi hanno realtà al di fuori della loro sintesi. A quei procedimenti astrattivi deve pertanto contrapporsi il criterio della forma come unità organica, che «non è un'idea, ma una cosa», e cioè la realtà stessa in quanto si configura nella mente dell'artista realizzandosi in un nuovo organismo, che è esso medesimo momento in sé perfetto ed insopprimibile del processo vitale. 
Se tuttavia il criterio della forma giova a stabilire l'autonomia e l'individualità del fenomeno artistico, come valore irriducibile di volta in volta al mutarsi e al progredire delle ideologie e delle tecniche, che cosa potrà ancora assicurare l'unità e la continuità di un discorso storico che si proponga di ritrovare un senso, una linea progressiva, nella serie dei fatti poetici come tali? Proprio, pensava il De Sanctis, la loro storicità intrinseca, la possibilità di un costante riferimento dialettico di quei fatti alla realtà, di cui essi sono al tempo stesso specchio e ricreazione ed elemento operante.

[...]

La critica è veramente tale solo se diventa storia, cioè se riesce ad inserirsi in una visione globale dello svolgimento storico. Per tale via, proprio mentre elaborava la critica più radicale e in qualche modo definitiva di ogni tipo di storia meramente sociologica dei fatti artistici, il De Sanctis si ingegnava (o s'illudeva?) di salvare l'idea di una storia della letteratura che, nella piena consapevolezza della relativa autonomia e individualità dei suoi oggetti, ritrovasse il filo di un discorso continuo e coerente riaffondando di volta in volta le radici nel terreno della storia civile e culturale della società.

A noi pare, e non soltanto per il prestigio ed il fascino dell'opera in cui egli si sforzò di porgere un primo tentativo di attuazione delle sue idee, che la soluzione del De Sanctis conservi intatta la sua validità anche oggi. ${ }^{21}$

E ancora, in chiara distinzione dalla ben nota condanna crociana delle storie letterarie a favore del saggio monografico, ma forse anche dell'ormai affermata critica stilistica e formale, Sapegno propone un vero e proprio capovolgimento di paradigma, in cui è semmai la critica letteraria, il saggio monografico di derivazione crociana, a mostrarsi inevitabilmente bisognoso di un retrostante disegno storiografico:

Come è noto, il problema di una giustificazione della storiografia letteraria ed artistica e di una distinta funzione nel quadro della storiografia generale, è tornato a porsi in tempi recenti con maggiore risolutezza, e anche questa volta proprio in Italia in termini più lucidi e rigorosi per merito soprattutto del Croce, il quale ha negato che si potesse postulare un complesso di caratteri comuni fra le singole opere di poesia, e quindi il criterio di una serie ideale oltre che cronologica, perché, egli diceva, «ogni carattere comune riferendosi alla materia delle opere artistiche (e materia è anche la loro forma quando è presa astrattamente e materializzata, il cosiddetto stile) non ha più valore estetico e non vale a congiungere fatti di natura estetica»: non si dà pertanto storia letteraria come narrazione di una tradizione organica e distinta di una serie di fatti omogenei; e ogni opera che si proponga come tale si risolve, nel migliore dei casi, in un seguito discontinuo di saggi monografici, malamente imprigionati in uno schema extraestetico. [...]

La condanna sembra senza appello, almeno in sede teorica. Né certo contro di essa possono aver forza i tentativi sempre risorgenti di sistemazione sociologica, che avviliscono le opere poetiche a documenti, ma neppure valgono gli opposti e altrettanto astratti tentativi di storicizzazione degli elementi

21. N. SAPEgnO, Introduzione alla Storia della Letteratura Italiana, I. Le Origini e il Duecento, Milano: 1965, p. viii-ix. 
tecnici rettorici e formali (del "gusto», delle "poetiche» o delle «strutture» polivalenti), elementi tutti che non hanno storia al di fuori dei singoli organismi poetici. Meno che mai potrebbero giovare a trarci d'impaccio le numerose storie che di fatto si continuano a scrivere della letteratura, della musica o delle arti figurative, le quali anzi spesso si potrebbe dire che confermino e ribadiscano in atto la condanna crociana, allorché appunto si riducono ad aggregati discontinui di monografie critiche collegate soltanto da un'esigenza didattica [...]. L'aporia, che sembra irriducibile, può esser superata soltanto ove si ritorni all'impostazione desanctisiana e si riconosca che i fatti artistici (ma non essi soli, anche i sistemi filosofici, i progressi gressi della scienza, gli eventi politici), mentre non si costituiscono in una serie autonoma e astrattamente riconoscibile in un ambito chiuso, crescono, e pertanto diventano oggetto di concreto studio, solo in quanto si collocano nel flusso totale delle condizioni storiche, in cui prendono il loro significato più vero anche i dati della tradizione letteraria e gli apporti e le innovazioni linguistiche tecniche e strutturali. Per questa via il problema potrà addirittura capovolgersi; se è vero che appare sempre più evidente l'improbabilità di dar fondamento di scienza a una critica letteraria che non si identifichi in una storia della letteratura, intesa come storia della civiltà nella particolare prospettiva delle vicende letterarie, e capace di riassorbire nel sentimento concreto e individualizzante dei valori poetici l'indagine genetica del complesso e multiforme contenuto che in quei valori si configura come in nuovi organismi e, mentre li determina, ne è a sua volta determinato nel suo progredire. ${ }^{22}$

Le posizioni di Dionisotti, note già dal 1951, non hanno corso nella prospettiva storiografica italiana almeno fino al 1967, quando egli pubblica, nella collana dei «Saggi» einaudiani, Geografia e storia della letteratura italia$n a$, appena due anni dopo l'uscita dell'imponente storia letteraria collettiva della Garzanti, ancora organizzata secondo un modello "per secoli» (Duecento, Trecento, ecc.), ossia di fatto, secondo un criterio cronologico lineare di tipo "generazionale». ${ }^{23} \mathrm{E}$ ' difficile non pensare al volume di Dionisotti come ad una sorta di risposta alla riproposizione di una storia «desanctisiana»: un agile veliero contrapposto a un potente bastimento, che tuttavia suggerisce un possibile cambiamento di rotta. La prolusione del 1949, Geografia e storia della letteratura italiana, che dà il titolo al volume del 1967, costituisce, quantomeno oggettivamente, una proposta alternativa a quanto si era fino allora, e ancora due anni prima, pensato e scritto in Italia in materia di storiografia letteraria: importante e criticamente incontrovertibile proprio perché basata su un esame storico fattuale, con Croce, della situazione storico politica e culturale italiana prima del 1860 e perché capace di inserire coscientemente tale analisi nel quadro politico-culturale dell'Italia del dopoguerra (così come contemporaneamente e significativamente aveva fatto Curtius in Germania con la sua Letteratura europea e Medio Evo latino, ragio-

22. Ibid., p. ix-xi.

23. Sulle possibili diverse concezioni e proposte del tempo storiografico cfr. R. ANTONELLI, Tempo e spazio nella storiografia letteraria, in La scrittura e la storia. Problemi di storiografia letteraria, a cura di A. Asor Rosa, Firenze: La Nuova Italia, 1995, p. 161-195. 
nando su un'altra crisi, particolarmente evidente dopo la seconda guerra mondiale, quella dell'Europa):

Certo è che mai come all'indomani di una disfatta militare e nel decorso di una crisi politica che hanno insidiato l'unità e l'esistenza stessa, come nazione e come stato, dell'Italia, si è sentito forte il bisogno di vedere con chiarezza in che modo e fino a che punto l'Italia sia stata a tutt'oggi fatta, ma il problema che si è posto nei suoi termini propri di storia politica, invade per un buon tratto la storia della letteratura, e sollecita l'attenzione degli studiosi di questa. Già è significativo il fatto che una storia della letteratura, quella ormai classica di Francesco De Sanctis, sia, credo, il solo libro che alla maggioranza degli Italiani abbia offerto e tuttavia offra una suggestiva rappresentazione e interpretazione unitaria della loro storia. Né occorre ricordare che su documenti letterari, da Dante al Manzoni, è principalmente fondata la tradizione unitaria in Italia. Occorre però forse precisare che questa tradizione [...] non risulta [...] da un intempestivo ideale politico [...] [ma] da un tempestivo e vittorioso ideale letterario, dal mito che la cultura italiana del Rinascimento creò e impose di una Italia risvegliatasi dal suo lungo e impotente sonno medievale non più donna di province ma pur sempre donna di una ineguagliata $\mathrm{e}$ forse ineguagliabile civiltà. ${ }^{24}$

La letteratura ha fondato la tradizione unitaria in Italia: dopo una «disfatta militare» che ha "insidiato l'unità e l'esistenza stessa, come nazione e come stato, dell'Italia» occorre «riconsiderare» come si sia «fatta» politicamente l'Italia, ma anche, per necessaria conseguenza, come sia stata «fatta» e interpretata la storia della letteratura italiana.

La Storia di De Sanctis, in quanto storia emblematica dell'unità d'Italia, assunta unanimemente quale modello di storia letteraria militante, appare a Dionisotti, nella Premessa e dedica scritta per il volume einaudiano del 1967, ormai inadeguata, sia per le premesse storiche da cui partiva, sia per le problematiche che la situazione storica contemporanea poneva:

Poiché la via di un impegno letterario insieme e politico, e di una interpretazione storica del passato in funzione del presente, può apparire in Italia, e di fatto è stata variamente e anche recentemente proposta, quasi una ripresa della tradizione risorgimentale, devo dire, o piuttosto ripetere, che non ho mai per parte mia pensato alla possibilità di una tale ripresa. Né sul piano dell'azione politica, né su quello della ricerca storica. Il debito che abbiamo contratto con gli uomini del Risorgimento è fuori discussione. Ma le difficoltà e pertanto i compiti nostri sono stati e sono tutt'altri.

Ogni grande impresa richiede grandi sacrifici. Né è raro il caso che i sacrifici risultino a distanza maggiori del previsto e mettano finalmente a nudo la debolezza dei vincitori o dei loro eredi. E' senza dubbio il caso dell'impresa risorgimentale. L'ultima guerra non è bastata a infrangere l'unità d'Italia, ma ha rimesso in questione la struttura che all'Italia unita era stata imposta. 
[...] Compito nostro era di mettere, per quanto potessimo, un qualche riparo alla rovina di ogni cosa intorno a noi e in noi. Sempre avevamo creduto all'unità, e però a una storia d'Italia e a una storia della letteratura italiana. Ma sempre anche avevamo dubitato della struttura unitaria, che nell'età nostra era giunta a fare cosí trista prova di sé, e però anche di quella corrispondente storia d'Italia e della letteratura italiana, che era stata prodotta nell'età risorgimentale. Né il fatto che, a differenza della storia politica, proprio la storia della letteratura prodotta allora avesse ottenuto dal De Sanctis la forma del capolavoro, poteva in alcun modo attenuare il dubbio. Quella storia splendidamente rappresentava l'istanza unitaria del Risorgimento e il decisivo apporto del Regno di Napoli alla causa dell'unità. Ma per ciò stesso e per i caratteri propri della storia del Regno, diversa affatto e appartata per lunghi tratti da quella del resto d'Italia, ci si poteva chiedere se essa anche non rappresentasse, coi suoi tratti semplici e decisi, la sopraffazione spiccia che ai fini dell'unità era stata imposta alle differenze reali e tradizionali della vecchia Italia. [...]

A chi oggi si volga dubitando indietro, giova riconsiderare lo sforzo che, come già s'è detto, fu compiuto nell'età postrisorgimentale, dal 1860 innanzi, per vedere piú chiaro nella struttura e storia della vecchia Italia prerisorgimentale. Anche e piú gioverà risalire all'età memore e presaga, che piú di ogni altra volle vedere chiaro, al Settecento. Là, fra il Gravina e il Tiraboschi, ancora sono le fondamenta solide di una storia della vecchia Italia. Là anche, e di là risalendo indietro, sono i fatti, gli uomini, i testi, che nessuna successiva descrizione o interpretazione può sostituire e che devono essere riconsiderati uno a uno. ${ }^{25}$

Riconsiderati uno a uno; ma non appunto secondo "uno schema storicogeografico unitario dei rari e indipendenti “mondi” poetici che la critica romantica era venuta scoprendo e colonizzando»: «le grandi figure tragiche dell'Inferno dantesco si incontravano, nel pensiero del De Sanctis, non con altre nel susseguente processo della poesia italiana, ma se mai, fuori d'Italia e a intervallo di secoli, con le grandi figure tragiche dello Shakespeare. [...] Il paragone veniva a farsi fra quel che l'Italia sembrava essere stata e quel che avrebbe potuto o dovuto essere, e, come accade, il secondo termine influiva e si imponeva sul primo. ${ }^{26}$

Insomma, potremmo commentare, non al modo della Storia della Letteratura Italiana Garzanti, né di altre che con quella si confrontarono nei decenni successivi, tutte degne e spesso importanti per nuovi singoli contributi, ma basati su un modello ormai arcaico, pur se occorre sottolineare come Carlo Muscetta, nel 1970, nella presentazione della sua Letteratura italiana, Storia e testi (il titolo riprende quello della serie ricciardiana e punta alla sintesi unitaria di rappresentazione storiografica e antologia), avvertisse che il paradigma ormai scricchiolava, citando esplicitamente una relazione di Dionisotti, ma non la Geografia e storia della letteratura italiana uscita ormai da tre anni e restando sostanzialmente fedele al modello desanctisiano, pur rivendicando un'attenzione al policentrismo della letteratura italiana che faceva risalire addirittura a Settembrini:

25. Ibid., p. 8, 9 e 11.

26. Ibid., p. 31. 
Queste pagine di «presentazione» erano già in corso di stampa col piano generale dell'opera, quando sono state rese pubbliche al Congresso dell'Associazione internazionale per gli studi di lingua e letteratura italiana tre relazioni di Carlo Dionisotti, Mario Sansone e Dante Isella, particolarmente stimolanti per i problemi che il nostro gruppo di lavoro ha avuto e continuerà ad avere presenti.

Attraverso le discussioni degli italianisti si sono puntualizzati importanti aspetti metodici nella «ricerca di un nuovo canone di interpretazione storica, o per dirla in termini alquanto ambiziosi, di una forma totale ed organica della nostra storia letteraria»: come ha detto Mario Sansone, che non ha mancato di aggiungere, con solenne onestà, un significativo ripudio della nota scepsi crociana sulla possibilità di una storia della letteratura. «Riteniamo (pur intendendone nel tempo, pienamente, le ragioni polemiche e teoriche) ormai inaccettabile il principio della dissoluzione della storia letteraria nelle costruzioni monografiche sui singoli poeti». Non meno fondamentali ci sono parse le due preoccupazioni emerse nelle parole di Carlo Dionisotti : la ricerca di una consapevolezza storica del presente e la necessità di guardarsi dal "puzzo» di vieti esclusivismi, angustie ideologiche e borie locali, quando si affrontano i rapporti tra culture e tradizioni regionali e municipali, e letteratura nazionale. Siamo tanto convinti che non si possa prescindere da tesi così vivacemente e autorevolmente sviluppate, che già prima che nel nostro gruppo si definisse l'impianto di quest'opera, non avevamo mai dimenticato (ogni volta che se ne presentasse l'occasione) il carattere policentrico della nostra letteratura e le diverse condizioni di sviluppo culturale delle nostre regioni durante le varie epoche. Tanto più che a questo tema metodologico avevamo appuntato lo sguardo fin dall'inizio della nostra edizione delle opere di Francesco De Sanctis, venti anni fa, confrontando l'impostazione data dal grande maestro irpino con quella di altri tentativi storiografici : «Settembrini intendeva [...] far giustizia ai contributi più importanti che dalle varie regioni erano venuti alla letteratura nazionale». E ciò conteneva un suggerimento metodico, che se fosse stato tenuto nel debito conto dagli storici letterari successivi, poteva portare ad una visione unitaria più realistica, più obiettiva. ${ }^{27}$

Bisognerà aspettare una decina d'anni e la Letteratura italiana Einaudi diretta da Alberto Asor Rosa, perché si prenda veramente in considerazione l'ipotesi dionisottiana, pur se il punto di riferimento iniziale e di confronto è sempre De Sanctis, abbandonato a favore di una possibile storiografia dei testi, "delle forme» ${ }^{28}$, ma soprattutto a favore di una struttura di tipo tematico:

Il «diagramma De Sanctis»... e il nostro.

Chiunque affronti un ragionamento sulla storia della letteratura italiana, è costretto a misurarsi con la grande opera di Francesco De Sanctis. [...] Tuttavia l'acuta consapevolezza della sua grandezza serve oggi soprattutto a disegnare, anche con malinconia, l'abisso che ci separa dalla sua prospettiva.

27. C. Muscetta, La letteratura italiana Storia e testi, I. 1 Il Duecento. Dalle Origini a Dante, 1970 , p. 7.

28. Letteratura italiana, diretta da A Asor Rosa, I. Il letterato e le istituzioni, Torino: Einaudi, 1982, p. 22-23. 
Il mondo che egli ci propone non c'interessa più; un mondo nuovo si dispiega ai nostri occhi, proprio quando volgiamo le vele ad allontanarci dal suo.

Pensate al paradosso contenuto nella Storia della letteratura italiana di De Sanctis. Essa è l'opera piú significativa dedicata a celebrare, attraverso la letteratura, la civiltà italiana moderna e la sua identità nazionale: pure, il diagramma, che il De Sanctis disegna, è quello di una decadenza. Questa decadenza comincia in limine, e precisamente quando, tra Dante da una parte e Petrarca e Boccaccio dall'altra, alla figura del poeta si sostituisce quella del letterato e dell'artista. Comincia lì la secolare scissione tra l'uomo e lo scrittore, tra la cosa e la forma, che solo a metà Settecento scrittori dotati di forte senso morale (Parini, Alfieri), cominciano [...] a risanare. La sensibilità di De Sanctis è per tanti aspetti cosí moderna (ricordiamo le splendide letture dei testi di Petrarca) da risarcire almeno in parte le durezze dello schema. Ma queste durezze restano, e sono per molti versi decisive: le incomprensioni verso due grandissimi come Machiavelli e Guicciardini ne sono la testimonianza. Bisogna dunque sostituire interi pezzi dello schema, invertire le ascisse del diagramma.

Se si parte da questi due presupposti — e cioè che 1) «La letteratura italiana non può essere associata alla storia etica e civile della nazione italiana (anche se ovviamente ha con essa rapporti)»; 2) «Non necessariamente la grande letteratura nasce da una grande vita morale»_, si può arrivare finalmente a capire che l'aspetto veramente progressivo della letteratura italiana, la sua autentica gloria in cospetto al mondo, è la creazione (a partire da Dante, non escluso) di quel gigantesco sistema delle forme, di quell'affascinante proposta di vita per mezzo di segni, che dispiega fra Trecento e Seicento, attraverso e anche nonostante le crisi sociali e politiche piú acute, la sua fase di maggiore produttività e prestigio europeo. ${ }^{29}$

$\mathrm{Al}$ «diagramma» di Dionisotti, contrapposto a quello di De Sanctis, è invece dedicata totalmente l'introduzione alla Storia e geografia della letteratura italiana ${ }^{30}$, la serie con cui la Letteratura italiana Einaudi risolve, in una serie concepita originariamente come esclusivamente tematica (iscritta in parte sotto il modello dell'Enciclopedia Einaudi iniziata nel 1977 e diretta da Ruggiero Romano, e in parte sotto la Letteratura europea e Medio Evo latino di Curtius), il problema di una storia della letteratura italiana integrata alla serie tematica, che già proponeva «implicitamente» un'immagine «radicalmente ribaltata del nostro passato letterario e della sua periodizzazione», fondato, almeno nella prospettiva del prefatore, più sulla coscienza della Krisis europea (e quindi su Curtius) che non sulle più recenti metodologie critiche (formalistiche e semiologiche, cui si riferiva invece Asor Rosa), pur esse conseguenza particolare della più generale Crisi della cultura umanistica:

Con questo volume inizia la parte conclusiva, dedicata —riprendendo una nota analisi di Carlo Dionisotti- alla Storia e geografia della letteratura italia-

29. Ibid.

30. R. ANTONELl, Storia e geografia, tempo e spazio nell'indagine letteraria, in Letteratura italiana, Storia e geografia I. L'età medievale, Torino: Einaudi, 1987, p. 5-26. 
na. La storia della letteratura "unilineare» (fondata su una successione cronologica ordinata di cause ed effetti, spesso "progressivi», posti sotto la regia di un unico principio ispiratore), viene sostituita da un modello articolato di realtà storico-geografiche, non esaminate in quanto semplici premesse, contorni o conferme della letteratura nazionale post-unitaria. Per di piú la stessa Storia e geografia è a sua volta concepita come un insieme «modulare» da porre in correlazione e in reazione con tutte le analisi «trasversali» contenute nei volumi della precedente sezione tematica.

La novità complessiva del progetto destò, alla pubblicazione del primo volume [...], molte e vive discussioni, legate al definitivo abbandono del modello storiografico prevalente, quello desanctisiano, e alla proposta di un diverso modo di fare storia letteraria e di concepire la letteratura, che teneva ormai conto, nell'assumere il testo a polo primario della ricerca, del dibattito novecentesco intorno alla teoria e ai metodi letterari, specie in ambito linguisticoformale e semiologico.

[...] Implicitamente, ma non tanto, i sei volumi già pubblicati della Letteratura italiana propongono un'immagine radicalmente ribaltata del nostro passato letterario e della sua periodizzazione. Nello stesso rifiuto della storia «cronologica» progressivamente ordinata e nella dissezione del materiale storico per tagli trasversali, uno dei punti focali dell'ellissi, quello decisivo, è consapevolmente piantato nel Novecento: è appunto il nostro presente, ivi compresa la sua progettualità di futuro. La periodizzazione conseguente riconosce e costituisce il sistema letterario italiano in un unico insieme, sia pure scandito da tempi interni. Se ci si fosse però limitati a questa quasi inevitabile constatazione si sarebbe proposta soltanto una versione lievemente aggiornata e forse banalizzata del nesso passato-presente, storia-politica, pensiero-azione. Il fatto è che tale periodizzazione non solo fa centro sulla soggettività situazionale e storico-politica degli autori e dei lettori della Letteratura italiana, ma identifica in tale soggettività l'esito nuovo radicalmente diverso, per quanto ancora fluido e transitorio, di un evento "catastrofico» e di una "crisi»: la perdita delle certezze e dell'oggettività dei Valori (e dei metodi) e la contemporanea nascita e sviluppo della moderna cultura di massa. La riaggregazione di tutto quanto precede in un sistema diverso e globalmente unitario ne è la logica conseguenza.

Potrà apparire ancora oggi sorprendente una concezione storiograficoletteraria che leghi in un unico blocco tutta la letteratura italiana (e occidentale), dalle origini delle moderne letterature volgari alla nascita e allo sviluppo della cultura di massa.

[...] È certo però che nella coscienza e nella prassi della parte piú significativa e alta della grande letteratura e critica europea è proprio tra la fine dell'Ottocento e gli anni '30 del Novecento, nel cuore del «moderno», che si situa la crisi che muta organicamente (e geneticamente) i termini stessi della letteratura e del "fare letterario", creativo e critico.

Senza le riflessioni che tale situazione nuova ha determinato nelle zone piú varie della letteratura e della cultura, ivi compresa quella apparentemente piú protetta e lontana, la storiografia e la teoria letteraria, è altrettanto certo che la Letteratura italiana Einaudi, in quanto struttura formale e metodologica e in quanto progetto di ricerca, non sarebbe nata o avrebbe comunque assunto forme molto diverse. 
La questione dunque del «modello De Sanctis» a questo punto non riguarda più immediatamente il versante politico-culturale (anche se in effetti si contrappone alla politica-culturale del PCI nel dopoguerra e negli anni Cinquanta), ma il modello storiografico e i suoi presupposti, certamente anch'essi latamente definibili come politico-culturali, come ogni atto storico-critico, ma innanzitutto teorici e storici: unitario/lineare (alla De Sanctis, o comunque al modo storicistico evenemenziale) o diacronico-tematico (alla Curtius) e storico-geografico (alla Dionisotti)? Riguarda, in definitiva, due diverse concezioni dello spazio e del tempo. Cosa sottende la concezione riflessa nella Letteratura Einaudi? Certamente la crisi dell'intellettuale post-'68 (la Letteratura Einaudi è messa in cantiere in un'altra data fatidica per l'Italia, il 1977) e il processo d'industrializzazione e "proletarizzazione» dei paesi a capitalismo avanzato: conseguentemente ha come punto di partenza il tema del Caos e della Krisis e le risposte date alla crisi dalla cultura europea sul piano interpretativo: dalla «longue durée» alle periodizzazioni e tematizzazioni lunghe, volte a capire le permanenze strutturali e a proiettarle sul futuro. Quanto a dire, come in Reinhart Koselleck, una riflessione sull' idea del tempo storiografico.

Negli anni più recenti si è tentato di ripristinare, talvolta anche per banali motivi di mercato editoriale, un modello storiografico più "semplice», fondato su uno storicismo lineare, non più desanctisiano, ma comunque di stampo originariamente ottocentesco: è il caso della Storia della letteratura italiana, diretta per le edizioni Salerno da Enrico Malato (iniziata nel 1995), dove peraltro, accanto alla consapevole ed esplicita ripresa della tradizione (tanto da far apparire «inopportuna un'ampia disquisizione teorica preliminare»), si dà spazio anche alla questione del policentrismo italiano e si allarga l'orizzonte alla storia delle istituzioni e alle connessioni fra letteratura e altre arti:

In questa prospettiva —qui appena schematizzata: ma è parsa inopportuna un'ampia disquisizione teorica preliminare, quasi a «giustificazione» del lavoro che si presenta- è stata giudicata, piú che possibile, necessaria una nuova Storia della Letteratura Italiana che, strutturata in modo "tradizionale», si distinguesse da altre esistenti per la diversa articolazione interna della materia e per il píu ampio orizzonte entro il quale i fatti letterari vengono indagati e ricostruiti. Avvicinandosi una scadenza — la fine del millennio — che sarà comunque un'occasione di consuntivi e di bilanci, mentre la suggestione delle mode ideologiche e l'esasperata ricerca del «nuovo» ha portato altrove alla decomposizione e di fatto alla dissoluzione del quadro storico, sempre piú pressante e perentoria si è avvertita l'esigenza di ricomporre quel quadro, di ridisegnare un diagramma lungo il quale potesse utilmente svilupparsi la ricerca sui testi e sui rispettivi percorsi, dalla produzione e circolazione alla ricezione e attualízzazione, intesi come "tracciato» dei percorsi della civiltà italiana.

[...] La Storia della Letteratura Italiana che qui si presenta va dunque intesa come «storia della civiltà letteraria» se non, tout court, come «storia della civiltà italiana». La storia letteraria viene ripercorsa sullo sfondo e nelle necessarie connessioni non soltanto - soprattutto in alcuni particolari momenti storici- con la storia della lingua, ma altresì con la storia dell'arte, la storia 
della musica, la storia del pensiero e in generale la storia della cultura, considerata anche nei suoi rapporti di scambio con le altre culture europee (e, quando sia il caso, extraeuropee), nonché con la storia dei fatti e delle istituzioni politiche, alla quale le vicende della cultura sono sempre levate e dalla quale sono spesso orientate e condizionate. Una specifica attenzione è riservata ai modi della tradizione e della ricezione dei testi e ai connotati regionali della letteratura italiana, recuperando se e quando possibile aspetti e momenti della cultura popolare accanto a quelli della tradizione aulica. ${ }^{31}$

Ma è il caso soprattutto della riduzione apportata alla Letteratura italiana Einaudi dallo stesso Asor Rosa per le edizioni pubblicate quali supplemento al quotidiano "La Repubblica», con eliminazione della serie dionisottiana della Storia e geografia della letteratura italiana, a vantaggio di una più consueta storia suddivisa per secoli, secondo il modello della Storia della letteratura italiana Garzanti 1965 e della Storia letteraria d'Italia della Casa editrice Vallardi 1926.

Pochi anni dopo, il modello dionisottiano sarà invece riproposto da Maria Serena Sapegno e da Roberto Antonelli in una storia e antologia, L'Europa degli scrittori, dedicata, come già quella di Natalino Sapegno, alle Scuole superiori italiane, dove anche la storia della letteratura europea viene riletta alla luce del modello "geografia e storia», tanto più in un momento di crisi dell'unità d'Italia e del processo d'integrazione europea:

Il titolo dell'opera sottolinea nel titolo il ruolo della letteratura nella formazione della coscienza, delle idee, dei valori e delle emozioni degli europei. Riconosce anche la stretta relazione fra letteratura europea e letteratura italiana e la funzione dell'Italia nella formazione di quel sistema letterario europeo che costituisce oggi (e costituirà ancor più nel prossimo futuro) l'orizzonte necessario di ogni riflessione sul senso della letteratura.

Non esiste ancora una «letteratura europea» condivisa e insegnata nelle scuole, ma esiste già una coscienza comune su quali possano essere i testi caratterizzanti, al di là di lingue e frontiere, di un "canone europeo", mentre di fatto esiste un canone della letteratura italiana, proposto innanzitutto attraverso la scuola. L'Europa degli scrittori pone perciò in relazione il canone della letteratura italiana con un possibile canone della letteratura europea, mostrando le dinamiche tra i centri e le periferie e le frequenti inversioni dei loro rispettivi ruoli: in alcuni periodi è infatti impossibile capire la letteratura italiana senza conoscere le opere della letteratura europea che ne costituiscono il presupposto (Origini, Illuminismo e Romanticismo, Novecento), mentre in altri (dalla fine del Duecento agli inizi del Seicento) è vero esattamente l'opposto. [...]

La critica riconosce già da decenni la validità del paradigma storiografico proposto da Carlo Dionisotti nel 1949 con Geografia e storia della letteratura italiana [...] senza che si sia tentato di trarne davvero le conseguenze sul piano formativo. Questa storia e antologia (come chiarisce il sottotitolo, Storia, centri, testi) presenta i testi collocandoli al'interno dei centri e degli Stati preuni-

31. Storia della letteratura italiana, diretta da E. Malato, I Dalle Origini a Dante, I. Le Origini, p. xii. 
tari, ovvero nel loro ambiente effettivo di produzione e fruizione, senza schiacciarli sulla ricostruzione compiuta dalla storiografia risorgimentale e postunitaria, ma senza sottovalutare l'importanza del ruolo svolto dalla lingua e dalla letteratura nella costruzione dell'identità italiana. Si è così rispettata, rendendola evidente, quella dialettica fra centri italiani (cittadini e regionalí), Stati nazionali ed Europa che costituisce oggi, come già in Italia al momento dell'Unità, un dato di fatto e insieme uno dei nodi problematici dell'unítà culturale e politica europea». ${ }^{32}$

Mai come in questo momento, a 150 anni dalla raggiunta unità d'Italia e in una nuova crisi europea, stavolta e per ora fortunatamente solo economica, è necessario riconsiderare, è necessario che soprattutto i giovani riconsiderino, per riprendere Dionisotti, "come e perché l'Italia e l'Europa siano state fatte» e quale ruolo possa giocare la letteratura in questa nuova situazione.

32. Roberto ANTONELli-Maria Serena SAPEGNO, L'Europa degli scrittori. Storia, centri, testi della letteratura italiana ed europea, 1a. Dalle origini al Trecento: la formazione del canone, p. iv-v. 